\title{
Les nématodes parasites associés au riz dans les trois (3) principales écologies rizicoles au Burkina Faso
}

\author{
Bouma THIO ${ }^{1 *}$, LEONARD S. OUEDRAOGO ${ }^{2}$, Elise SANON ${ }^{3}$, Philippe SANKARA ${ }^{3}$ et \\ Salam KIEMDE ${ }^{2}$
}

1 Institut de l'Environnement et de Recherches Agricoles (INERA), Département Production Végétale, Programme Riz et Riziculture, INERA-Kamboinsé,01 BP 476 Ouagadougou 01, Burkina Faso.

2 Institut de l'Environnement et de Recherches Agricoles (INERA), Département Production

Végétales,Programme Cultures Maraîchères, Fruitières et Plantes à Tubercules, INERA-Farako-Bâ, BP 910

Bobo-Dioulasso, Burkina Faso.

3 Université de Ouaga I Pr Joseph Ki-Zerbo, Unité de Formation et de Recherche/Science de la Vie et de la Terre (UFR/SVT), Laboratoire de Biosciences03 BP 7021 Ouagadougou 03, Burkina Faso.

*Auteur correspondant; E-mail: thiobouma@gmail.com

\section{RESUME}

Un inventaire des nématodes parasites du riz au Burkina Faso a été réalisé au cours de la campagne agricole humide 2016-2017. Ce travail de recherche vise à étudier la prévalence et l'abondance des principaux nématodes parasites associés au riz dans les 3 principales écologies rizicoles que sont la riziculture pluviale stricte, la riziculture de bas-fond et la riziculture irriguée. Pour cela, 156 échantillons de racines de riz et de sols attenantsont été prélevés dans 40 sites de production de riz; les nématodes ont été extraits du sol et des racines. Les résultats montrent quatorze (14) genres de nématodes parasites. Hirschmanniella et Tylenchorhynchus ont été fréquents et abondants dans les écologies rizicoles de bas-fond et irriguée car observés dans plus de $50 \%$ des échantillons de riz à des niveaux de populations supérieures à 200 nématodes $/ \mathrm{dm}^{3}$ de sol et 20 nématodes $/ \mathrm{g}$ de racines. Tylenchorhynchus, Helicotylenchus, Scutellonema ont été observés en riziculture pluviale stricte. Meloidogyne, Heterodera et Pratylenchus ont été recensés sur le riz dans les écologies pluviales strictes et basfond. Ces 7 groupes de nématodes peuvent être considérés comme les plus dommageables au riz dans les écologiesoù ils sont importants.

(C) 2017 International Formulae Group. All rights reserved.

Mots clés: Nématodes parasites, riz, écologie rizicole, Hirschmanniella, Tylenchorhynchus, Burkina Faso.

\section{Plant parasitic nematodes associated to rice in the three (3) main riceecologies of Burkina Faso}

\begin{abstract}
A survey of rice parasitic nematodes has been conducted in Burkina Faso during the rainy crop season 2016-2017. This research aimedat studying the occurrence and abundance of rice parasitic nematodes in the 3 main rice ecologies (upland rainfed, lowland and irrigated rice). For that, 156 samples of roots and adjacent soils were collected at 40 locations of rice production. The extraction of nematodes was done
\end{abstract}


according to the methods of Seinhorst $(1950,1962)$. The results showed that 14 genera of parasitic nematodes were identified. Hirschmanniella and Tylenchorhynchus were frequent and abundant in lowland and irrigated rice ecologies because observed in more than $50 \%$ of the samples with nematode population levels higher to 200 nematodes $/ \mathrm{dm}^{3}$ of soils and 20 nematodes/g of roots. Tylenchorhynchus, Helicotylenchus and Scutellonema were observed in upland rice. Meloidogyne, Heterodera and Pratylenchus were observed in the lowland and upland rice ecologies. These 7 groups of nematodes can be considered as the most damaging to rice in these ecologies and are susceptible to cause significant damages if the conditions are good for their multiplication.

(C) 2017 International Formulae Group. All rights reserved.

Keywords: Rice parasitic nematodes, rice ecology, Hirschmanniella, Tylenchorhynchus, Burkina Faso.

\section{INTRODUCTION}

Le riz occupe la $4^{\text {ème }}$ place parmi les céréales cultivées au Burkina Faso après le sorgho, le maïs et le mil. La production de riz a progressé de plus de $50 \%$ en six ans, passant 195.000 tonnes en 2008 à 305.000 tonnes en 2013 grâce aux mesures de soutien suite à crise céréalière de 2008 (FAO, 2014). Néanmoins, la production nationale de riz couvre moins de $50 \%$ des besoins de consommation, d'où des importations massives qui sont passées de 255.000 tonnes en 2008 et 440.000 tonnes en 2013 (FAO, 2014).

Les sorties en devises pour l'importation du riz pour la même période sont passées de 40,9 milliards de FCFA en 2008 à près de 62 milliards en 2013 (FAO, 2014). La demande nationale de riz en 2018 pourrait atteindre 466.000 tonnes de riz usiné, soit plus de 600.000 tonnes de riz paddy au regard de l'évolution de la population et de la consommation nationale annuelle (FAO, 2014). En effet, la consommation annuelle par tête d'habitant est passée de $18 \mathrm{~kg}$ en 1999 à $35 \mathrm{~kg}$ en 2013 (Rapport CPSA-DGESS (2013) cité par FAO (2014)). Elle atteint actuellement plus de $50 \mathrm{~kg}$ par personne et par an dans les centres urbains de Ouagadougou et de BoboDioulasso (FAO, 2014).

Le Burkina Faso dispose d'un potentiel important non encore exploité dans le domaine de la riziculture avec des superficies potentiellement irrigables estimées à 233.500 ha (MAHRH, 2004). Quant aux basfonds aménageables, le potentiel est estimé à environ 1,9 millions d'hectares dont 500.000 hectares facilement aménageables avec $10 \%$ actuellement mis en valeur (BM-SP-CPSA, 2012).

La riziculture de bas-fond est la forme traditionnelle de riziculture la plus observée car pratiquée dans toutes les zones agroclimatiques du pays. Elle est réalisée dans des sites sans maitrise de l'eau (bas-fonds traditionnels non aménagés) ou dans des sites avec maîtrise partielle de l'eau (bas-fonds aménagés simples ou bas-fonds améliorés). Avec $67 \%$ des superficies totales exploitées en riz, les bas-fonds fournissent $42 \%$ de la production nationale en riz, avec un rendement moyen variant de $1,3 \mathrm{t} / \mathrm{ha}$ (basfonds non-aménagés) à 2,5 t/ha (bas-fonds aménagés) avec un potentiel de $4 \mathrm{t} / \mathrm{ha}$ pour les bas-fonds aménagés (CNRST, 2005).

Quant à la riziculture irriguée avec maîtrise totale de l'eau, le pays dispose d'un potentiel important non encore exploité avec des superficies potentielles irrigables estimées à 233.500 hectares. C'est le mode de production de riz le plus performant en raison de la maîtrise totale de l'eau permettant la double campagne annuelleavec des rendements de 4 à 7 tonnes/ha. La riziculture irriguée occupe en moyenne $23 \%$ des superficies rizicoles et fournit près de $53 \%$ de la production nationale en riz (CNRST, 2005).

Enfin, la riziculture pluviale stricte reste encore très marginale et est tributaire de la quantité et de la répartition des pluies; elle est pratiquée dans les régions du Burkina Faso où la pluviométrie dépasse $800 \mathrm{~mm} / \mathrm{an}$. Elle occupe $10 \%$ des superficies dévolues au riz et fournit $5 \%$ de la production nationale en riz 
avec un rendement moyen de 1t/ha (CNRST, 2005).

L'augmentation de la production nationale de riz est donc un enjeu stratégique et des efforts sont développés afin de réduire l'importation croissante du riz.

Parmi les défis majeurs d'amélioration des rendements et de l'accroissement des productions figure la lutte contre les bioagresseurs du riz, dont les nématodes parasites du riz. Bridge et al. (2005) ont signalé que les nématodes parasites du riz constituent des contraintes biotiques majeures pour le riz et sont capables de causer des pertes de rendement et affecter la qualité des semences. Aux Philippines, les nématodes les plus observés dans les écologies rizicoles de bas-fonds sont $M$. graminicola, Hirschmanniella oryzae et $H$. mucronata; les autres nématodes sont représentés par Aphelenchoides, Pratylenchus, Tylenchorhynchus, Helicotylenchus, Criconemoides, and Rotylenchus (Pascual et al., 2014). Les pertes de rendement des cultures causées par les nématodes parasites sont estimées à 14,6\% dans les pays tropicaux et subtropicaux et $8,8 \%$ dans les pays développés (Nicol et al., 2011); elles sont estimées à 10\% pour le riz (Agrios, 2005).

L'objectif de cette étude est de caractériser les communautés de nématodes parasites associés au riz dans les différentes écologies rizicoles du Burkina Faso afin de connaître leur importance et de développer ultérieurement des méthodes de lutte efficaces, améliorer les rendements et augmenter durablement la production de riz.

\section{MATERIEL ET METHODES}

L'étudesur les principaux nématodes parasites associés au riz a couvert les trois (3) zones agroclimatiques du Burkina Faso qui comprennent la zone sahélienne avec une pluviométrie annuelle inférieure à $600 \mathrm{~mm}$, la zone soudano-sahélienne avec une pluviométrie annuelle variant entre 600 et $900 \mathrm{~mm}$ et la zone soudanienne avec une pluviométrie annuelle comprise entre 900 et $1200 \mathrm{~mm}$. Le climat du Burkina Faso est caractérisé par l'alternance d'une saison humide et d'une saison sèche. La saison humide débute généralement au mois de mai dans la partie sud et s'achève en octobre.

Un total de cent cinquante-six (156) échantillons composites de racines et de sols attenants ont été prélevés dans 40 sites de production de riz dans les 3 écologies rizicoles à travers le Burkina Faso (Figure 1). Vingtquatre (24) échantillons ont été prélevés dans 6sites sur le riz pluvial strict dont la production reste limitée essentiellement dans l'Ouest du Burkina Faso. Soixante (60) et 72 échantillons ont été prélevés respectivement dans 20 et 18 sites sur le riz de bas-fond et le riz irrigué qui constituent les écologies rizicoles les plus dominantes. Deux (2) sites ont été concernés par des prélèvements d'échantillons dans les écologies rizicoles pluviales strictes et bas-fond.

L'échantillon composite est constitué par 5 prélèvements de racines et de sols attenants. Trois (3) échantillons composites ont été prélevés par champ principalement au stade grain laiteux - début maturité.

\section{Traitement des données}

Après dénombrement, le nombre de nématodes a été rapporté au $\mathrm{dm}^{3}$ de sol humide pour les nématodes exophytes et au gramme de racine sèche pour les nématodes endophytes.

L'importance de chaque genre et espèce de nématode a été déterminée à partir du Diagramme de Fréquence/Abondance de Fortuner et Merny (1973).

Pour ces auteurs, la Fréquence (F) est le pourcentage d'échantillons qui contiennent le nématode considéré; elle est calculée par la formule suivante:

$$
\mathbf{F}=\mathbf{e} / \mathbf{n} .100
$$

Où : $\mathbf{e}=$ nombre d'échantillons contenant le genre considéré et $\mathbf{n}$ : nombre total d'échantillons.

L'Abondance (A) d'un genre de nématode est la moyenne des densités des échantillons dans lesquels le genre a été retrouvé. Elle est calculée sous la formule suivante:

$$
\mathbf{A}=\sum \mathbf{X i} / \mathbf{e}
$$


Où: $\mathbf{X i}=$ nombre d'individus du genre considéré par $\mathrm{dm}^{3}$ de sol ou par gramme de racines sèches et $\mathbf{e}=$ nombre d'échantillons dans lesquels le genre considéré est présent. Ce paramètre est en logarithme décimal.

Ces auteurs considèrent qu'un genre est abondant et fréquent quand il est présent dans au moins 30\% des échantillons avec au moins 200 individus par $\mathrm{dm}^{3}$ de sol ou 20 individus par gramme de racines sèche. En dessous de ces valeurs, il est considéré peu fréquent et peu abondant. Par contre, un genre de nématode peut être considéré fréquent et peu abondant ou alors peu fréquent et abondant.

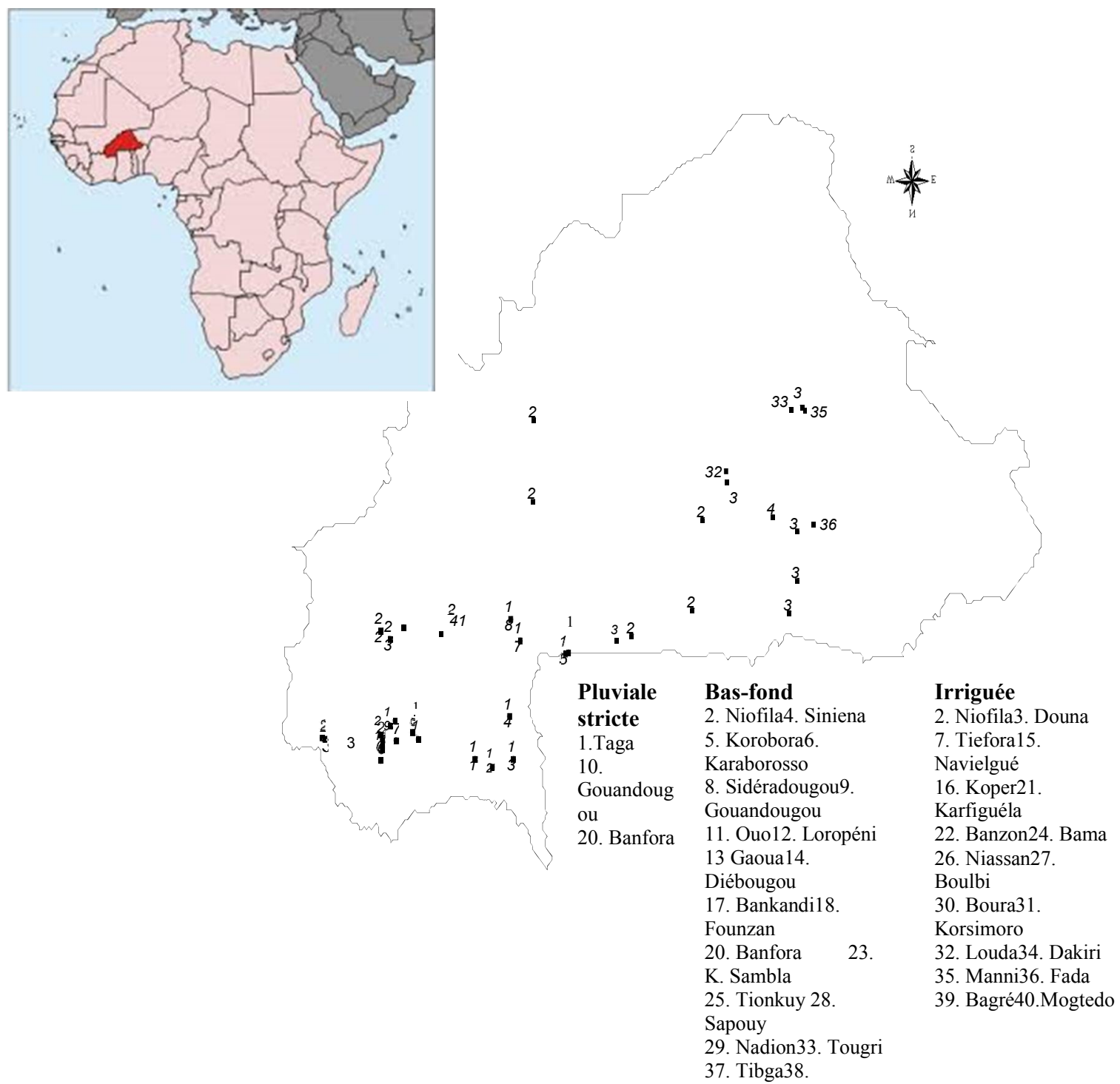

Figure 1: Sites de prélèvement des échantillons de racines et sols pour l'étude de la prévalence des nématodes parasites du riz au Burkina Faso. 


\section{RESULTATS}

Prévalence et abondance des communautés de nématodes parasites associés au riz au Burkina Faso

Quatorze (14) genres de nématodes parasites sont recensés sur le riz au Burkina Faso, avec une diversité importante des communautés selon le type de riziculture (Tableau 1). Certains genres et espèces apparaissent plus inféodés au riz de bas-fond et irrigué alors que d'autres semblent plus adaptés aux conditions de terres hautes de riziculture pluviale stricte.

\section{En riziculture pluviale stricte}

Dix (10) genres/espèces de nématodes parasites phytoparasites ont été recensés. Trois genres apparaissent fréquents et abondants; il s'agit de Scutellonema (dans 92\% des échantillons), Tylenchorhynchus (92\%), Helicotylenchus (88\%) (Tableau 1A). Les nématodes Pratylenchus, Criconemoides et Paratrichodorus ont été fréquents mais peu abondants avec moins de 200 individus par $\mathrm{dm}^{3}$ de sol. Les nématodes appartenant aux genres Telotylenchus, Paratylenchus, et Xiphinema apparaissent peu abondants (moins de 200 individus par $\mathrm{dm}^{3}$ de sol) et peu fréquents à des niveaux inférieurs à $30 \%$. Meloidogyne a été peu fréquent mais observé à des niveaux de populations élevées. Les nématodes $H$. spinicaudata, $H$. oryzae, Heterodera, Hoplolaimus et $A$. besseyi n'ont pas été observés dans cette écologie.

Deux genres de nématodes (Meloidogyne et Pratylenchus) ont été extraits des racines et ont été abondants (plus de 20 individus/g de racines) mais peu fréquents car trouvés dans respectivement 12 et $4 \%$ des échantillons (Figure 1B).

\section{En riziculture de bas-fond}

Le riz de bas-fond apparaît le plus infesté par les nématodes parasites avec la totalité des nématodes parasites, observés sur le riz au Burkina Faso, soit 14 genres. Tylenchorhynchus et Hirschmanniella spinicaudata ont été les plus abondants et les plus fréquents car présents respectivement dans $88 \%$ et $52 \%$ des échantillons (Figure
2A). Les nématodes Meloidogyne, Heterodera, Pratylenchus, H. oryzae, Aphelenchoides besseyi, Criconemoides sont abondants mais peu fréquents (Figure 2A). Les autres nématodes parasites ont été peu abondants et peu fréquents; il s'agit de Helicotylenchus, Scutellonema, Xiphinema, Paratrichodorus et Hoplolaimus. Pratylenchus, Helicotylenchus et Xiphinema. Telotylenchus et Paratrichodorus n'ont pas été observés.

Pour les nématodes extraits des racines de riz, deux espèces ont surtout été observées; il s'agit $H$. spinicaudata qui apparaît fréquente (dans $52 \%$ des échantillons) et abondante et l'espèce voisine $H$. oryzae est peu fréquente ( $5 \%$ des échantillons) et peu abondante (Figure 2B).

\section{En riziculture irriguée}

Douze (12) de genres de nématodes ont été trouvés. Les nématodes fréquents et abondants sont représentés par Tylenchorhynchus et $H$. spinicaudata car présents dans respectivement $79 \%$ et $67 \%$ des échantillons et à des niveaux de populations supérieures à 200 nématodes $/ \mathrm{dm}^{3}$ de sol (Figure 3A). H. oryzae, Helicotylenchus et Scutellonema apparaissent abondants mais peu fréquents car présents dans moins de $30 \%$ des échantillons (Figure 3A). Les autres nématodes sont observés à des niveaux de fréquences faibles et à des densités de populations faibles; il s'agit de Pratylenchus, Scutellonema, Criconemoides, A. besseyi et Meloidogyne. Les nématodes Heterodera, Paratrichodorus, Telotylenchus, Hoplolaimus, Paratylenchus n'ont été observés ou à des niveaux de populations très faibles (proches de 0).

$\mathrm{Au}$ niveau des populations de nématodes observés dans les racines, seule l'espèce H.spinicaudata est fréquente et abondante (Figure 3B). Les autres groupes de nématodes sont peu fréquents et peu abondants. Il s'agit de $H$. oryzae, A. besseyi, Pratylenchus, Meloidogyne et Heterodera (Figure 3B). 

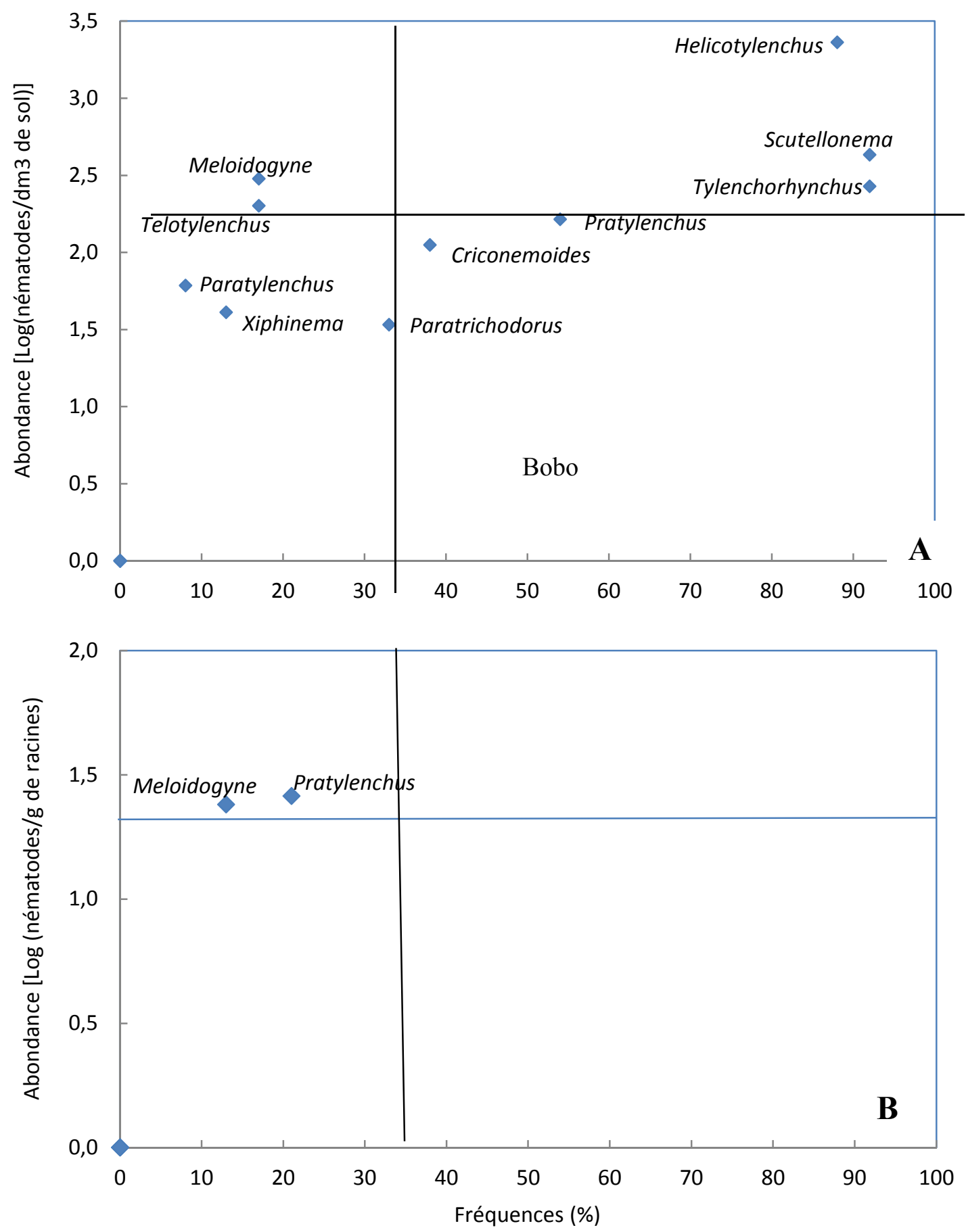

Figure 1 : Fréquence et abondance des genres et espèces de nématodes parasites du riz pluvial strict observés dans le sol (A) et dans les racines (B) selon la méthode de Fortuner et Merny (1973). 

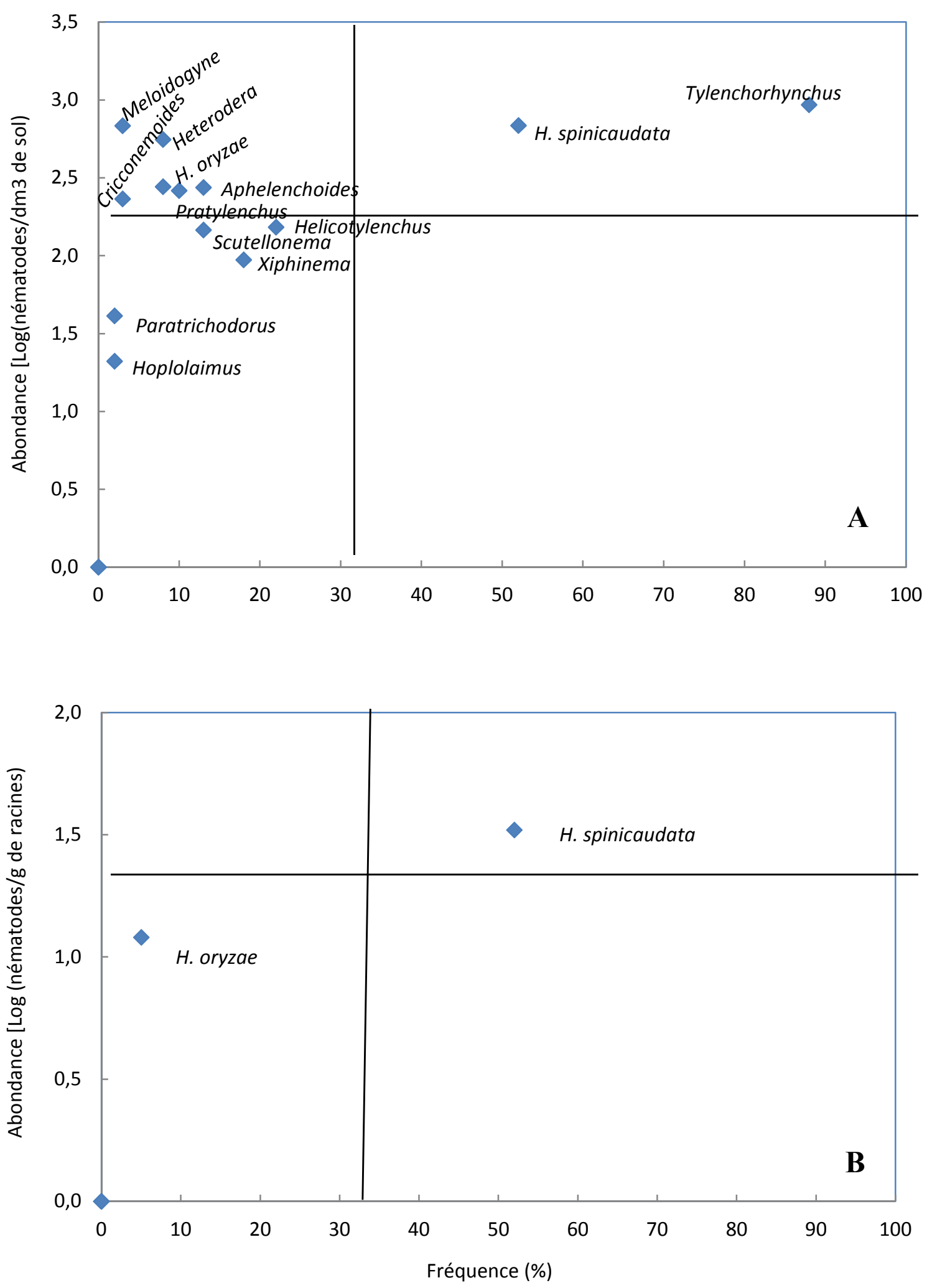

Figure 2: Fréquence et abondance des genres et espèces de nématodes parasites du riz de bas-fond observés dans le sol (A) et dans les racines (B) selon la méthode de Fortuner et Merny (1973). 

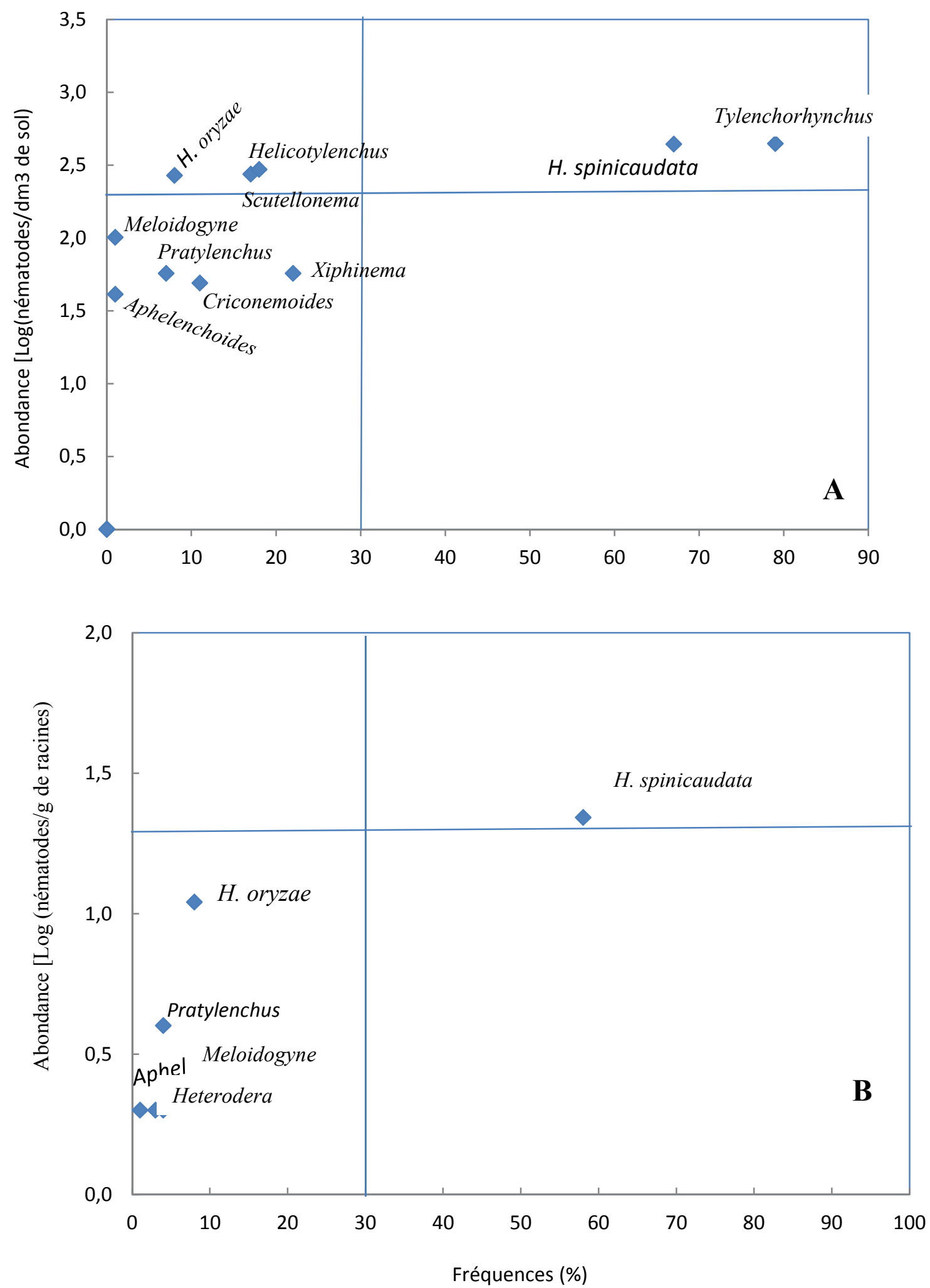

Figure 3 : Fréquence et abondance des genres et espèces de nématodes parasites du riz irrigué observés dans le sol (A) et dans les racines (B) selon la méthode de Fortuner et Merny (1973). 
Tableau 1: Fréquence d'observation des principaux genres et espèces de nématodes parasites du riz présents dans les échantillons de sols et de racines en fonction desécologies rizicoles du Burkina Faso.

\begin{tabular}{|c|c|c|c|c|c|c|}
\hline \multirow{3}{*}{$\begin{array}{c}\text { Genres et espèces de } \\
\text { nématodes }\end{array}$} & \multicolumn{6}{|c|}{ Ecologies rizicoles } \\
\hline & \multicolumn{2}{|c|}{ Pluviale stricte } & \multicolumn{2}{|c|}{ Bas-fond } & \multicolumn{2}{|c|}{ Irriguée } \\
\hline & Sols & Racines & Sols & Racines & Sols & Racines \\
\hline H. spinicaudata & - & - & $* *$ & $* *$ & $* *$ & $* *$ \\
\hline H. oryzae & - & - & $*$ & $*$ & $*$ & $*$ \\
\hline Heterodera & $*$ & $*$ & $*$ & $*$ & $*$ & $*$ \\
\hline Meloidogyne & $*$ & $*$ & $*$ & $*$ & * & $*$ \\
\hline Pratylenchus & $* *$ & $*$ & $*$ & - & $*$ & $*$ \\
\hline Tylenchorhynchus & $*$ & - & $* * *$ & - & $* *$ & - \\
\hline Helicotylenchus & $* * *$ & - & $*$ & - & $*$ & - \\
\hline Scutellonema & $* * *$ & - & $*$ & - & $*$ & - \\
\hline Xiphinema & $*$ & - & $*$ & - & $*$ & - \\
\hline Criconemoides & $* *$ & - & $*$ & - & $*$ & - \\
\hline Paratrichodorus & $* *$ & - & $*$ & - & - & - \\
\hline Telotylenchusannulatus & $*$ & - & $*$ & - & - & - \\
\hline Hoplolaimusrobustus & - & - & $*$ & - & - & - \\
\hline Paratylenchus & $*$ & - & - & - & - & - \\
\hline Aphelenchoidesbesseyi & - & - & $*$ & - & $*$ & - \\
\hline
\end{tabular}

\section{DISCUSSION}

Diversité des communautés de nématodes parasites du riz

La forte diversité des communautés de nématodes parasites observés dans l'écologie bas-fondpeut s'expliquer par sa typologie particulière car pouvant regrouper les 3 écologies rizicoles. Pascual et al. (2014) ont observé 5 genres de nématodes dans l'écologie bas-fond et 9 genres dans l'écologie pluviale stricte au Brésil. Au Burkina Faso, le bas-fond comprend,généralement, dans sa partie la plus haute, l'écologie pluviale stricte (riz de plateau) rarement soumis à une inondation et dans sa partie la plus basse, l'écologie irriguée soumise à une inondation généralement permanente pendant tout le cycle de développement du riz; la partie intermédiaire ou zone hydromorphe à texture et structure particulières en termes de sols est généralement soumise à une forte variabilité de la nappe phréatique influençant la diversité et les densités des peuplements de nématodes parasites pendant le cycle du riz.

Prévalence et importance des nématodes parasites du riz dans les écologies rizicoles

Nos résultats corroborent avec ceux de plusieurs travaux de recherches sur les communautés de nématodes parasites du riz.

En effet, plusieurs recherches ont noté la prédominance des nématodes appartenant au genre Hirschmanniella connus comme les plus fréquents et les plus dommageables au riz dans les écologies de bas-fond et irriguée (Coyne et al., 2000; Ravichandra et al., 2003; Bridge et al. 2005; Maung et al., 2010; Udo et al., 2011). L'inventaire des nématodes parasites associés au riz irrigué au Burkina Faso a montré que $H$. oryzae était surtout observée présente en zone sahélienne; en soudanienne l'espèce était observée en 
populations mixtes avec $H$. spinicaudata (Sawadogo et al., 1994). Des pertes de rendement de 10 à $30 \%$ causées par $H$. oryzaeont été signalées en Chine (Liao et al., 2000).

Tylenchorhynchus est commun au riz produit dans toutes les écologies rizicoles (Bridge et al., 2005). Heterodera est surtout un parasite important du riz pluvial strict et de bas-fond (Bridge et al., 2005). Une réduction de la teneur en chlorophylle des feuilles de $50,4 \%$ a été notée sur le riz inoculé avec 10.000 œufs de Heterodera sacchari (larves de $2^{\text {ème }}$ stade) en pot et de $60,3 \%$ au champ naturellement infesté (Akpheokhai et al., 2014). Helicotylenchus est très présent sur le riz pluvial (Bridge et al., 2005) et Helicotylenchus dihystera a été observée en riziculture pluviale stricte et sur les parties hautes des bas-fonds de Côte d'Ivoire (Coyne et al., 1998). Pratylenchus est surtout inféodé aux cultures de terres hautes dont le riz de plateau (pluviale stricte); il est considéré comme le nématode parasite le plus fréquent dans cette écologie (Bridge et al., 2005; Pascual et al., 2014).

Meloidogyne est observé sur le riz dans le sud-est du Nigeria (Udo et al., 2011) et généralement dans les écologies pluviales et bas-fond (Bridge et al., 2005). Les résultats de recherches ont montré que les faibles populations de Meloidogyne ont été observées sur les sols sablo-limoneux à faible capacité de rétention en eau (parcelles rarement inondées) dans les écologies rizicoles de basfond et irriguée du Burkina Faso; les systèmes de production à base de riz associent comme plantes de rotations, les cultures telles la patate douce et le maïs plus sensibles. Meloidogyne observés en fortes infestations sur le riz est connu causer des réductions significatives sur le développement des plants se traduisant par une réduction de la hauteur, du nombre de talles, du poids de la matière sèche, du poids des racines et de la teneur en chlorophylle des feuilles (Bimpong et al., 2010). Plusieurs auteurs ont noté des gains de rendement de $12-80 \%$ par le contrôle de
Meloidogyne spp. sur le riz produit en conditions aérobies ou inondées de façon temporaire (Padgham et al., 2004; Soriano et Reversat, 2003).

Enfin, plusieurs autres nématodes ont été observés dans les 3 écologies rizicoles mais et leurs faibles fréquence et abondance ne permettent pas de dégager leur l'importance en termes de pathogénicité sur le riz. C'est le cas de Criconemoidesonoensis qui provoque un rabougrissement et un jaunissement des plants dans des tests en pots avec des lésions observées sur les extrémités des racines secondaires (Chinapen et al., 1988). Quant au genre Xiphinema, il se nourrit sur la partie supérieure des racines et est reconnu comme pouvant transmettre des virus (Demangeat, 2007).

\section{Conclusion}

Les nématodes appartenant aux genres Hirschmanniella et Tylenchorhynchus apparaissent comme les plus fréquents et les plus dommageables au riz dans les écologies rizicoles de bas-fond et irriguées. Tylenchorhynchus, Helicotylenchus, Scutellonemas ont plus inféodées au riz pluvial strict. D'autres groupes de nématodes sont recensés dans les écologies pluviales et bas-fond et peuvent causer des dommages importants si les conditions sont favorables à leur multiplication. Il s'agit Meloidogyne, Heterodera et Pratylenchus.

Ces groupes de nématodes peuvent être considérés comme les plus dommageables au riz au Burkina Faso et devront faire l'objet de recherches de méthodes de lutte.

\section{CONFLIT D'INTERETS}

Il n'existe pas de conflit d'intérêts entre les différents auteurs de cet article.

\section{CONTRIBUTIONS DES AUTEURS}

BT a conduit les travaux de recherches sur le terrain, avec l'appui technique de SK et rédigé l'article. Les contributions de BT et SK peuvent être estimées respectivement à 50 et 
$10 \%$. Les autres auteurs ont contribution à la lecture et à la correction de l'article et leur contribution peut être estimée à $40 \%$.

\section{REMERCIEMENTS}

Les auteurs tiennent à remercier le Ministère en charge de l'Agriculture, notamment la Direction Générale des Productions Végétales (DGPV) et le Projet Riz Pluvial pour les appuis apportés dans la réalisation de cette étude.

\section{REFERENCES}

Agrios GN. 2005. Parasitism and Disease Development. In Plant Pathology ( $5^{\text {th }}$ edn). Elsevier Academic Press: UK; 901.

Akpheokhai LI, Fawole B, Claudius-Cole AO. 2014. Effects of Heterodera sacchari on Leaf Chlorophyll Content and Root Damage of some Upland NERICA Rice Cultivars. Journal of Agriculture and Veterinary Science, 7(8): 49-57.

Bimpong IK, Carpena AL, Mendioro MS, Fernandez L, Ramos J, George Reversat G, Darshan S, Brar DS. 2010. Evaluation of Oryza sativa x O. glaberrima derived progenies for resistance to rootknot nematode and identification of introgressed alien chromosome segments using SSR markers. African Journal of Biotechnology, 9(26): 3988-3997. DOI: 10.5897/AJB2010.000-3275

BM-SP-CPSA. 2012. Revue diagnostique des dépenses publiques de base dans le secteur agricole (2004-2012): Projet de document de travail spécifique à l'aménagement hydro-agricole. Banque Mondiale, Secrétariat Permanent pour la Coordination des Politiques Sectorielles Agricoles.

Bridge J, Plowright RA, Peng D. 2005. Nematode Parasite of Rice. In Plant Parasitic Nematodes in Subtropical and Tropical Agriculture, Luc M, Sikora RA,
Bridge J (eds). CAB International: Wallingford, U.K; 87-130.

Chinappen M, Lamberti F, Ciancio A, Jokun P. 1988. Losses caused by concomitant infestations of Criconomellaonoensis and Helicotylenchus dibystera on upland rice in Mauritius. Nematol. Medit., 16: 175-177.

Coyne DL, Plowright RA, Thio B, Hunt DJ. 1998. Plant parasitic nematode diversity and prevalence in traditional upland rice in Ivory Coast: preliminary observations on the effects of cropping intensification. Fundam. Appl. Nematol., 21(6): 723732.

Coyne DL, Hunt DJ, Plowright RA, Darboe MLK. 2000. Further observations on nematodesassociated with rice in Côte d' Ivoire, The Gambia, Guinea and Togo. International Journal of Nematology, 2(10) : 123-130.

Centre National de la Recherche Scientifique et Technologique (CNRST). 2005. Le riz au Burkina Faso in Eurêka spécial Riz Septembre 2005, 17-25.

Demangeat D. 2007. Transmission des Nepovirus par les nématodes Longidoridae. Virologie, 11(4): 309-321. DOI : 10.1684/vir.2007.0102

FAO. 2014. Analyse des incitations par les prix pour riz au Burkina Faso. Série de notes techniques, Rome, 61p.

Fortuner R, Merny G. 1973. Les nématodes parasites des racines associés au riz en Basse Casamance (Sénégal) et en Gambie. Cah. ORSTOM, Sér. Biol., 21: 3-20.

Liao JL, Feng ZX, Li SH, Hu YM. 2000. Species of Hirschmanniella on rice and their distribution in China. Nematologia Mediterranea, 28:107-110.

MAHRH. 2004. Politique nationale de développement durable de l'agriculture irriguée: stratégie, plan d'action et plan d'investissement, horizon 2015 (PNDDAI). Ministère de l'Agriculture, 
de l'Hydraulique et des Ressources Halieutiques.

MAHRH. 2010. Stratégie Nationale de Développement de la Riziculture (SNDR), Ministère de l'Agriculture, de l'Hydraulique et des Ressources Halieutiques, Ouagadougou, Burkina Faso, P. 43.

Maung ZTZ, Kyi PP, Myint YY, Lwin T, de Waele D. 2010. Occurrence of the rice root nematode Hirschmanniella oryzae on monsoon rice in Myanmar. Tropical Plant Pathology, 35(1): 003-010. DOI: 10.1590/S1982-56762010000100001

Nicol JM, Turner SJ, Coyne DL, Nijs L. Den Hockland S, Thana Maafi Z. 2011. Current nematodes threats to world agriculture. In Genomics and Molecular Genetics of Plant-Nematode Interactions Jones J, Gheysen G, Fenoll C (eds). Springer: Dordrecht; 21-43. DOI: 10.1007/978-94-007-0434-3.

Padgham JL, Duxbury JM, Mazid AM, Abawi GS, Hossain M. 2004. Yield losses caused by Meloidogyne graminicola on lowland rainfed rice in Bangladesh. $J$. Nematol., 36: 42-48. DOI: 10.1080/03235408.2011.588059

Pascual MLD. Decraemer W, Tandingan De Ley I, Vierstraete A, Steel H, Bert W. 2014. Prevalence and characterization of plant-parasitic nematodes in lowland and upland rice agro-ecosystems in Luzon, Philippines. Nematropica, 44: 166-180.

Ravichandra NG, Krishnappa K, Reddy BMR. 2003. Occurrence and distribution of phytoparasitic nematodes associated with rice in Mandya District, Karnataka. Indian Journal of Nematology, 33: $178 . \quad$ DOI: $\quad 10.1590 /$ S198256762010000100001

Sawadogo A, Thio B, Abdoulaye H, Konaté A. 1994. Inventaire des nématodes associés au riz irrigué au Sahel du Burkina et à l'Office du Niger au Mali. Nuisibles Pests-Pragas, 2: 130-148.

Seinhorst JW. 1950. De betekenis van de grond voor het optreden van aantasting door het stengelaaltje (Ditylenchus dipsaci) (Kühn Filipjev). Tijdschr. Plantenzietkten, 56: 289-348.

Seinhorst JW. 1962. Modifications of the elutriation method for extracting nematodes from soils. Nematologica, 8: 117-128.

Soriano IR, Reversat G. 2003. Management of Meloidogynegraminicolaand yield of upland rice in South-Luzon (Philippines). Nematology, 5: 879-884.

Udo IO, Nneke NE, Uyioata AM. 2011. Survey of plant parasitic nematodes associated with rice (Oryza sativa L.) in South Eastern Nigeria. African Journal of Plant Science, 5(10): 617-619. 